\title{
Monitoring patient health based on medical records using fuzzy logic method
}

\author{
Mawaddah Harahap \\ Universitas Prima Indonesia \\ mawaddah@unprimdn.ac.id
}

\author{
Ari Rizky Pratama \\ Universitas Prima Indonesia \\ arierpratama.official@gmail.com
}

\author{
Wali Siregar \\ Universitas Prima Indonesia \\ walisiregar@gmail.com
}

\author{
Delano Ariesagita Hutagalung \\ Universitas Prima Indonesia \\ delanohutagalung978@gmail.com \\ Hendra Sihombing \\ Universitas Prima Indonesia \\ $\underline{\text { hendrasihombing48@gmail.com }}$
}

\begin{abstract}
The Fuzzy Logic concept was first introduced as a way of processing data by allowing the use of partial set membership compared to the crisp set membership or non-membership. Along with the development of computer technology, the concept of fuzzy logic is increasingly needed by people, because this concept is able to provide information needed in the decision making process. This study aims to analyze and design intelligent systems to monitor the health development of inpatients. The method used is the Fuzzy Logic method. This method will predict the level (degree) of health of each patient based on the amount of drug use and the duration of diagnosis process. The tools used to analyze and design the system are Unified Modeling Language. The results of this research are monitoring the health development of patients using the Fuzzy Logic method.
\end{abstract}

Keywords: Fuzzy Tsukamoto, patient health monitoring, medical records

\section{INTRODUCTION}

In almost every aspect of life, humans are always faced with several choices. Making the right decisions will be very influential in our lives going forward. Artificial Intelligence is one of the fields of computer science that uses computers so that they can behave intelligently like humans. Computer Science is developing software and hardware to be able to imitate the actions of humans. Artificial intelligence can solve problems by using computers to solve complex problems by following human reasoning processes [1].

Research in the field of health and pharmacy has been carried out before, such as research on the design of an integrated service post information system at the Surabaya-based Web City Health Office [2] which produces a Posyandu information system that provides convenience in running information systems (user friendly) and convenience and speed in making Posyandu reports. Research on System Strategy and Information Technology Planning at Rs. Damian Lewoleba With Ward And Peppard's Approach [3] which discusses the application of technologies such as networks, databases, and platforms. Other research on Productivity Using Nurse Working Time in Inpatient Installation of Ahmad Yani Hospital Pekanbaru [4] which produced information in the form of nurses' productive work time ie $42.4 \%$ (i.e. $19.6 \%$ direct activities and indirect nursing activities $22.8 \%$ ) and non-productive $57.6 \%$, so it can be concluded that the productivity of the use of nurse's work time is not optimal and is more dominated by nonproductive activities such as: talking outside the main tasks, watching TV, using gadgets or going out for other activities. However, some of these studies do not discuss the development of patient health. 
Research on the fuzzy logic method has also been carried out by several previous researchers, such as research on Fuzzy Logic Analysis Determining the Selection of Honda Motors with the Mamdani Method [5], obtaining information that the process of Matlab Beat motor shows $23.3 \%$ and defuzzification results of manual Beat motorbike shows 24,049\%, the process from Matlab motor Vario shows $79.4 \%$ and the defuzzification result of manual Vario motor count shows $83.88 \%$, the process from Supra motor matlab shows $23.2 \%$ and the defuzzification result of manual Supra motor shows $21.049 \%$. so this result shows the Vario motorbike to be bought. Other research concerning the Application of Fuzzy Logic Method to Evaluate Nurse Service Performance (Case Study: RSIA Siti Hawa Padang) [6] who obtained information that the fuzzy logic method for evaluating nurse performance in hospitals, according to the desired results and fuzzy logic methods can be used to nurse performance assessment.

To solve the problems faced in several previous studies, then in this study Fuzzy Logic algorithm will be used to analyze and monitor the health development of patients. The Fuzzy Logic concept was first introduced by Prof. Lotfi Zadeh from the University of California at Berkeley in 1965, and presented not as a control methodology, but as a way of processing data by allowing the use of partial set membership compared to the crisp set membership or non-membership. Along with the development of computer technology, the concept of fuzzy logic is increasingly needed by people, because this concept is able to provide information needed in the decision making process.

With the design of the system, it is expected to benefit the hospital, especially regarding monitoring the health development of patients at the hospital. This eventually became a challenge for the author to make a study into a research entitled Monitoring the development of patient health based on medical records using the fuzzy logic algorithm.

\subsection{Smart System}

Smart systems are AI programs (Artificial Intelligence) or artificial intelligence that combine the knowledge base with inference machines. This is a part of high-level specialization software or high level language. Intelligent systems can adopt a small part of the level of human intelligence, among others, the ability to be trained, recalling conditions that have been experienced, processing data to provide appropriate action according to what has been taught, and the ability to absorb the expertise of an expert through commands written in a certain programming language.

\subsection{Fuzzy Logic Method}

Fuzzy Logic is an appropriate way to map an input space into an output space. The starting point of the modern concept of uncertainty is the paper made by Lofti A Zadeh (1972), where Zadeh introduced a theory that has objects from fuzzy sets that have limits that are not precise and membership in fuzzy sets, and not in the form of true logic ( true) or false, but expressed in degrees.

Fuzzy logic is an increase of Boolean logic that deals with partial concepts of truth. When classical logic states that everything can be expressed in binary terms ( 0 or 1 , black or white, yes or no) fuzzy logic replaces Boolean truth with a level of truth. The set of fuzzy logic was first introduced by Lotfi A. Zadeh in 1965 as a mathematical way to represent linguistic uncertainty. Based on the concept of fuzzy logic, factors and criteria can be classified without binding restrictions. Fuzzy logic is very useful for resolving high problems in various fields which usually contain degrees of uncertainty. The theory of set fuzzy logic was developed by Professor Lofti A. Zadeh in 1965. He argues that right and wrong logic of conventional Boolean logic cannot overcome the problem of gradation in the real world. To overcome this infinite gradation problem, Zadeh developed a fuzzy set. Unlike boolean logic, fuzzy logic has continuous value. Fuzzy is expressed in degrees of membership and degrees of truth. Therefore something can be said to be partially true and partly wrong at the same time. Based on the above, fuzzy logic can be used to model a mathematical problem, where the mathematical concepts underlying fuzzy reasoning are very simple and easy to understand.

Logic is only a small part of the capacity of the human mind for a reason. Logic can be a means to force us to conclude the right answer, but it cannot by itself be responsible for our creativity or for our ability to remember. In other words, logic can help us arrange words to make clear sentences, but cannot help us determine what sentences to use in various contexts. Consider the top part of nineteenth century mathematics Lewis Carroll in the Classic Through the Looking Glass. How high can we see the logical context in this fictional character's discourse? Logic for humans is a quantitative way to develop a reasoning process that can be replicated and 
manipulated with mathematics. Interest in logic is the study of truth in logical propositions, in the classic logic of this truth is binary - propositions are true or false. From this perspective, fuzzy logic is a method for formalizing human capacity right reasoning, or approximate reasoning. reasoning like this is a human ability to reason less and judge under uncertainty. in fuzzy logic all truths are only partial or approximate. In this case, this reason has also been called reasoning interpolation, where the process of interpolating between extreme binary from right and wrong is represented by the ability of fuzzy logic to summarize partial truths. [16]

\subsection{Fuzzy Tsukamoto Method}

"Fuzzy Inference System is a computational framework based on fuzzy set theory, fuzzy rules in the form of IF-THEN, and fuzzy reasoning".

Broadly speaking, block diagrams are fuzzy inference processes as below (Figure 1).

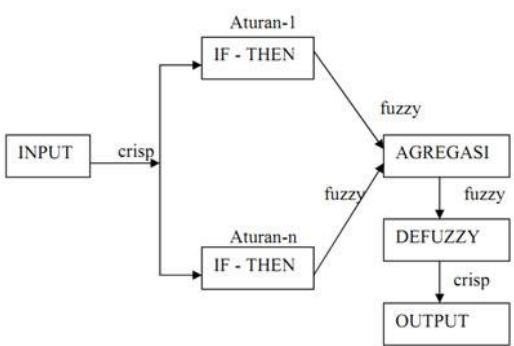

\section{Figure 1. Block Diagram of Fuzzy Inference} System

Fuzzy inference systems accept crisp input. This input is then sent to the knowledge base containing $n$ fuzzy rules in the IF-THEN form. Fire strength will be searched for in each rule. If the number of rules is more than one, then all rules will be aggregated. Furthermore, the results of the aggregation will be defuzzy to get the crisp value as the system output.

Block diagram of the inference process using the Tsukamoto method as below (Figure 2).

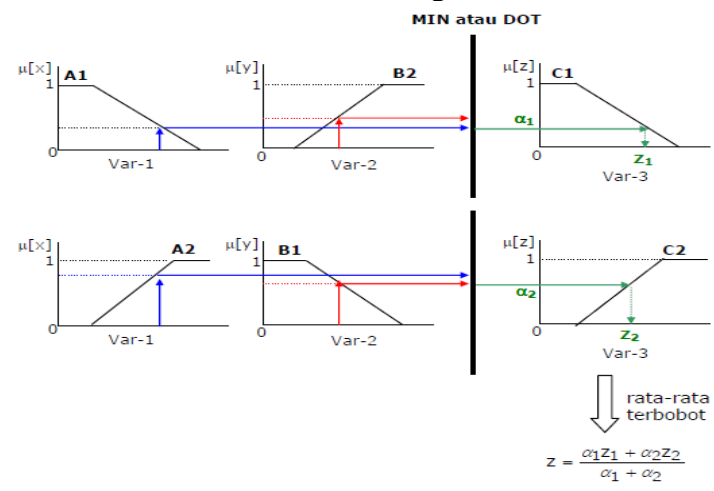

\section{Figure 2. Inference Process Block Diagram Using} Tsukamoto Method

Basically, the Tsukamoto method applies monotonous reasoning to each rule. In monotonous reasoning, the system has only one rule, in the Tsukamoto method, the system consists of several rules. Because it uses the basic concept of monotonous reasoning, in the Tsukamoto method, every consequence of IF-THEN rules must be represented by a fuzzy set with a monotonous membership function. The output of the inference results from each rule is given explicitly (crisp) based on $\alpha$-predicate (fire strength). The process of aggregation between rules is done, and the final results are obtained by using defuzzy with the concept of weighted averages. Suppose there are input variables, namely $\mathrm{x}$ and $\mathrm{y}$, and one output variable, $\mathrm{z}$. The variable $\mathrm{x}$ is divided into 2 sets, namely $\mathrm{A} 1$ and $\mathrm{A} 2$, the $\mathrm{y}$ variable is divided into 2 sets as well, namely B1 and B2, while the output variable $\mathrm{Z}$ is divided into 2 sets, namely $\mathrm{C} 1$ and $\mathrm{C} 2$. Of course the set $\mathrm{C} 1$ and $\mathrm{C} 2$ must be a monotonous set. 2 rules are given as follows:

$$
\begin{aligned}
& \text { IF } x \text { is } A 1 \text { and } y \text { is } B 2 \text { THEN } z \text { is } C 1 \\
& \text { IF } x \text { is } A 2 \text { and } y \text { is } B 2 \text { THEN } z \text { is } C 1
\end{aligned}
$$

\subsection{Data Analysis}

Analysis is an activity that starts from the initial process in learning and evaluating a form of problem that exists. The distribution of analysis, namely analysis of input documents, process analysis, and analysis of output documents.

The data sources used in this study are patient data and patient disease diagnosis data from one of the private hospitals in 2016.

The data obtained from hospitals in 2016 after passing the data selection process for this study are as follows:

a. Patient Data

Patient data was obtained from patient registration in 2016. These patient data consisted of patient attributes_id, age_group and gender. The patient data used can be seen in the table below:

Table 1. Patient Data Pasien for 2016 Year

\begin{tabular}{|l|l|l|}
\hline $\begin{array}{l}\text { Patient_I } \\
\text { D }\end{array}$ & $\begin{array}{l}\text { Age } \\
\text { Group }\end{array}$ & Gender \\
\hline P1 & Lansia & $\begin{array}{l}\text { Perempu } \\
\text { an }\end{array}$ \\
\hline P2 & Lansia & $\begin{array}{l}\text { Perempu } \\
\text { an }\end{array}$ \\
\hline P3 & Lansia & $\begin{array}{l}\text { Perempu } \\
\text { an }\end{array}$ \\
\hline
\end{tabular}




\begin{tabular}{|l|l|l|}
\hline P4 & $\begin{array}{l}\text { Dewas } \\
\text { a }\end{array}$ & $\begin{array}{l}\text { Laki- } \\
\text { Laki }\end{array}$ \\
\hline P5 & Lansia & $\begin{array}{l}\text { Laki- } \\
\text { Laki }\end{array}$ \\
\hline$\ldots$ & $\begin{array}{l}\text { Dewas } \\
\text { a }\end{array}$ & $\begin{array}{l}\text { Laki- } \\
\text { Laki }\end{array}$ \\
\hline P65535
\end{tabular}

b. Diagnosis Master Data

This Diagnosis Master Data consists of the types of illnesses suffered by patients based on the doctor's diagnosis obtained from the patient's medical record in 2016. Diagnosis data consists of the diagnosis_code attribute, diagnosis name. The diagnostic data used can be seen in the table below:

Table 2. Diagnosis Table for 2016 Year

\begin{tabular}{|c|}
\hline Diagnosis_Name \\
\hline Pra Hipertensi \\
\hline Hipertensi1 \\
\hline Hipertensi2 \\
\hline Hipotensi \\
\hline
\end{tabular}

c. Diagnosis Data based on Age and Gender Diagnosis data based on age and gender is obtained from the patient's disease diagnosis data and the age and sex of patients diagnosed with the disease. Diagnosis data consists of diagnostic attributes, code, diagnosis, name, group, name, gender. The diagnostic data used can be seen in the table below:

Table 3. Diagnosis Table Based on Age and

\begin{tabular}{|c|c|c|c|c|}
\hline $\begin{array}{c}\text { Pa } \\
\text { tie } \\
\text { nt }\end{array}$ & $\begin{array}{c}\text { Age } \\
\text { (old }\end{array}$ & $\begin{array}{c}\text { Gend } \\
\text { er } \\
\text { (P/L) }\end{array}$ & $\begin{array}{c}\text { Blood } \\
\text { Pressure } \\
\text { (mmHg) }\end{array}$ & Item \\
\hline $\mathbf{1}$ & 44 & $\mathrm{~L}$ & 80 & $\begin{array}{c}\text { dewasa, laki- } \\
\text { laki, hipotensi }\end{array}$ \\
\hline & & & 135 & $\begin{array}{c}\text { lansia, } \\
\text { perempuan, } \\
\text { prahipertensi }\end{array}$ \\
$\mathbf{2}$ & 79 & $\mathrm{P}$ & 145 & $\begin{array}{c}\text { dewasa, } \\
\text { perempuan, } \\
\text { hipertensi1 }\end{array}$ \\
\hline $\mathbf{3}$ & 43 & $\mathrm{P}$ & 143 & $\begin{array}{c}\text { dewasa, laki- } \\
\text { laki, } \\
\text { hepertensi1 }\end{array}$ \\
\hline $\mathbf{4}$ & 51 & $\mathrm{~L}$ & 143 & $\begin{array}{c}\text { dewasa, laki- } \\
\text { laki, }\end{array}$ \\
\hline $\mathbf{5}$ & 60 & $\mathrm{~L}$ & 149 & \\
\hline
\end{tabular}

\begin{tabular}{|c|c|c|c|c|}
\hline & & & & hipertensi1 \\
\hline 6 & 55 & $\mathrm{~L}$ & 161 & $\begin{array}{c}\text { dewasa, laki- } \\
\text { laki, } \\
\text { hipertensi2 }\end{array}$ \\
\hline 7 & 35 & $\mathrm{P}$ & 163 & $\begin{array}{l}\text { dewasa, } \\
\text { perempuan, } \\
\text { hipertensi2 }\end{array}$ \\
\hline 8 & 50 & $\mathrm{~L}$ & 166 & $\begin{array}{c}\text { dewasa, laki- } \\
\text { laki, } \\
\text { hipertensi2 }\end{array}$ \\
\hline 9 & 74 & $\mathrm{P}$ & 74 & $\begin{array}{c}\text { lansia, } \\
\text { perempuan, } \\
\text { hipotensi }\end{array}$ \\
\hline 10 & 33 & $\mathrm{~L}$ & 135 & $\begin{array}{l}\text { dewasa, laki- } \\
\text { laki, } \\
\text { prahipertensi }\end{array}$ \\
\hline 11 & 40 & $\mathrm{~L}$ & 170 & $\begin{array}{c}\text { dewasa, laki- } \\
\text { laki, hipetensi2 }\end{array}$ \\
\hline 12 & 49 & $\mathrm{~L}$ & 134 & $\begin{array}{l}\text { dewasa, laki- } \\
\text { laki, } \\
\text { hipertensi1 }\end{array}$ \\
\hline 13 & 57 & $\mathrm{P}$ & 83 & $\begin{array}{c}\text { dewasa, } \\
\text { perempuan, } \\
\text { hipotensi }\end{array}$ \\
\hline 14 & 56 & $\mathrm{P}$ & 84 & $\begin{array}{c}\text { dewasa, } \\
\text { perempuan, } \\
\text { hipotensi }\end{array}$ \\
\hline 15 & 45 & $\mathrm{P}$ & 142 & $\begin{array}{c}\text { dewasa, } \\
\text { perempuan, } \\
\text { hipertensi2 }\end{array}$ \\
\hline 16 & 71 & $\mathrm{~L}$ & 146 & $\begin{array}{c}\text { lansia, laki- } \\
\text { laki, } \\
\text { hipertensi1 }\end{array}$ \\
\hline 17 & 67 & $\mathrm{~L}$ & 136 & $\begin{array}{l}\text { lansia, laki- } \\
\text { laki, } \\
\text { prahipertensi }\end{array}$ \\
\hline 18 & 78 & $\mathrm{~L}$ & 75 & $\begin{array}{c}\text { lansia, laki- } \\
\text { laki, hipotensi }\end{array}$ \\
\hline 19 & 62 & $\mathrm{~L}$ & 144 & $\begin{array}{l}\text { lansia, laki- } \\
\text { laki, } \\
\text { hipertensi1 }\end{array}$ \\
\hline 20 & 59 & $\mathrm{~L}$ & 135 & $\begin{array}{l}\text { dewasa, laki- } \\
\text { laki, } \\
\text { prahipertensi }\end{array}$ \\
\hline 21 & 52 & $\mathrm{~L}$ & 159 & $\begin{array}{c}\text { dewasa, laki- } \\
\text { laki, } \\
\text { hipertensi1 }\end{array}$ \\
\hline 22 & 72 & $\mathrm{~L}$ & 83 & $\begin{array}{c}\text { lansia, laki- } \\
\text { laki, hipotensi }\end{array}$ \\
\hline 23 & 78 & $\mathrm{~L}$ & 133 & $\begin{array}{l}\text { lansia, laki- } \\
\text { laki, } \\
\text { prahipertensi }\end{array}$ \\
\hline
\end{tabular}




\begin{tabular}{|c|c|c|c|c|c|c|c|}
\hline \multirow[b]{2}{*}{24} & \multirow[b]{2}{*}{68} & \multirow{2}{*}{$\mathrm{L}$} & & \multirow[b]{3}{*}{56} & \multirow[b]{3}{*}{56} & \multirow[b]{3}{*}{$\mathrm{P}$} & \multirow{3}{*}{$\begin{array}{c}\text { sedang, normal } \\
\text { dewasa, perempuan, } \\
\text { sedang, normal }\end{array}$} \\
\hline & & & laki, normal & & & & \\
\hline$\ldots$ & $\ldots$ & $\ldots$ & $\ldots$ & & & & \\
\hline \multirow{2}{*}{\multicolumn{4}{|c|}{$\begin{array}{l}\text { Table 4. Diagnosis Table based on Age, Gender, } \\
\text { Duration of Diagnosis and Tension }\end{array}$}} & 57 & 54 & $\mathrm{P}$ & $\begin{array}{l}\text { dewasa, perempuan, } \\
\text { cepat, normal }\end{array}$ \\
\hline & & & & 58 & 63 & $\mathrm{~L}$ & $\begin{array}{l}\text { lansia, laki-laki, } \\
\text { sedang, normal }\end{array}$ \\
\hline $\begin{array}{l}\text { Pati } \\
\text { ent }\end{array}$ & $\begin{array}{l}\text { Age } \\
\text { (Old) }\end{array}$ & $\begin{array}{c}\text { Gender } \\
(\mathrm{P} / \mathrm{L})\end{array}$ & Item & 59 & 72 & $\mathrm{P}$ & $\begin{array}{l}\text { lansia, perempuan, } \\
\text { sedang, normal }\end{array}$ \\
\hline 35 & 69 & $\mathrm{~L}$ & $\begin{array}{l}\text { lansia, laki-laki, } \\
\text { sedang, lemah }\end{array}$ & 60 & 75 & $\mathrm{P}$ & $\begin{array}{c}\text { lansia, perempuan, } \\
\text { lama, normal }\end{array}$ \\
\hline 36 & 87 & $\mathrm{P}$ & $\begin{array}{l}\text { lansia, perempuan, } \\
\text { sedang, normal }\end{array}$ & 61 & 57 & $\mathrm{P}$ & $\begin{array}{c}\text { dewasa, perempuan, } \\
\text { cepat, normal }\end{array}$ \\
\hline 37 & 69 & $\mathrm{P}$ & $\begin{array}{l}\text { lansia, perempuan, } \\
\text { sedang, normal }\end{array}$ & 62 & 53 & $\mathrm{P}$ & $\begin{array}{c}\text { dewasa, perempuan, } \\
\text { sedang, lemah }\end{array}$ \\
\hline 38 & 50 & $\mathrm{~L}$ & $\begin{array}{c}\text { dewasa, laki-laki, } \\
\text { cepat, normal }\end{array}$ & 63 & 66 & $\mathrm{P}$ & $\begin{array}{c}\text { lansia, perempuan, } \\
\text { cepat, normal }\end{array}$ \\
\hline 39 & 51 & $\mathrm{P}$ & $\begin{array}{c}\text { dewasa, perempuan, } \\
\text { lama, normal }\end{array}$ & 64 & 63 & $\mathrm{P}$ & $\begin{array}{c}\text { lansia, perempuan, } \\
\text { sedang, lemah }\end{array}$ \\
\hline 40 & 47 & $\mathrm{~L}$ & $\begin{array}{l}\text { dewasa, laki-laki, } \\
\text { sedang, lemah }\end{array}$ & 65 & 53 & $\mathrm{P}$ & $\begin{array}{l}\text { dewasa, perempuan, } \\
\text { sedang, lemah }\end{array}$ \\
\hline 41 & 49 & $\mathrm{~L}$ & $\begin{array}{c}\text { dewasa, laki-laki, } \\
\text { sedang, normal }\end{array}$ & 66 & 37 & $\mathrm{~L}$ & $\begin{array}{c}\text { dewasa, laki-laki, } \\
\text { cepat, normal }\end{array}$ \\
\hline 42 & 59 & $\mathrm{P}$ & $\begin{array}{l}\text { dewasa, perempuan, } \\
\text { sedang, normal }\end{array}$ & 67 & 63 & $\mathrm{~L}$ & $\begin{array}{c}\text { lansia, laki-laki, cepat, } \\
\text { normal }\end{array}$ \\
\hline 43 & 64 & $\mathrm{P}$ & $\begin{array}{l}\text { lansia, perempuan, } \\
\text { sedang, normal }\end{array}$ & 68 & 75 & $\mathrm{P}$ & $\begin{array}{c}\text { lansia, perempuan, } \\
\text { cepat, normal }\end{array}$ \\
\hline 44 & 59 & $\mathrm{~L}$ & $\begin{array}{c}\text { dewasa, laki-laki, } \\
\text { sedang, normal }\end{array}$ & 69 & 69 & $\mathrm{~L}$ & $\begin{array}{c}\text { lansia, laki-laki, cepat, } \\
\text { normal }\end{array}$ \\
\hline 45 & 75 & $\mathrm{~L}$ & $\begin{array}{l}\text { lansia, laki-laki, } \\
\text { sedang, lemah }\end{array}$ & 70 & 72 & $\mathrm{~L}$ & $\begin{array}{l}\text { lansia, laki-laki, } \\
\text { sedang, lemah }\end{array}$ \\
\hline 46 & 57 & $\mathrm{P}$ & $\begin{array}{l}\text { dewasa, perempuan, } \\
\text { sedang, normal }\end{array}$ & 71 & 60 & $\mathrm{~L}$ & $\begin{array}{c}\text { dewasa, laki-laki, } \\
\text { sedang, lemah }\end{array}$ \\
\hline 47 & 64 & $\mathrm{P}$ & $\begin{array}{l}\text { lansia, perempuan, } \\
\text { lama, normal }\end{array}$ & 72 & 35 & $\mathrm{~L}$ & $\begin{array}{l}\text { dewasa, laki-laki, } \\
\text { sedang, lemah }\end{array}$ \\
\hline 48 & 63 & $\mathrm{~L}$ & $\begin{array}{c}\text { lansia, laki-laki, cepat, } \\
\text { normal }\end{array}$ & 73 & 57 & $\mathrm{~L}$ & $\begin{array}{c}\text { dewasa, laki-laki, } \\
\text { sedang, normal }\end{array}$ \\
\hline 49 & 63 & $\mathrm{P}$ & $\begin{array}{l}\text { lansia, perempuan, } \\
\text { sedang, normal }\end{array}$ & 74 & 57 & $\mathrm{~L}$ & $\begin{array}{c}\text { dewasa, laki-laki, } \\
\text { cepat, normal }\end{array}$ \\
\hline 50 & 59 & $\mathrm{P}$ & $\begin{array}{c}\text { dewasa, perempuan, } \\
\text { lama, lemah }\end{array}$ & 75 & 63 & $\mathrm{~L}$ & $\begin{array}{c}\text { lansia, laki-laki, cepat, } \\
\text { normal }\end{array}$ \\
\hline 51 & 73 & $\mathrm{~L}$ & $\begin{array}{l}\text { lansia, laki-laki, } \\
\text { sedang, normal }\end{array}$ & 76 & 55 & $\mathrm{~L}$ & $\begin{array}{c}\text { dewasa, laki-laki, } \\
\text { sedang, normal }\end{array}$ \\
\hline 52 & 47 & $\mathrm{~L}$ & $\begin{array}{c}\text { dewasa, laki-laku, } \\
\text { sedang, normal }\end{array}$ & 77 & 71 & $\mathrm{~L}$ & $\begin{array}{l}\text { lansia, laki-laki, } \\
\text { sedang, normal }\end{array}$ \\
\hline 53 & 75 & $\mathrm{~L}$ & $\begin{array}{l}\text { lansia, laki-laki, } \\
\text { sedang, normal }\end{array}$ & 78 & 77 & $\mathrm{P}$ & $\begin{array}{c}\text { lansia, perempuan, } \\
\text { cepat, normal }\end{array}$ \\
\hline 54 & 60 & $\mathrm{P}$ & $\begin{array}{l}\text { dewasa, perempuan, } \\
\text { sedang, normal }\end{array}$ & 79 & 58 & $\mathrm{~L}$ & $\begin{array}{l}\text { dewasa, laki-laki, } \\
\text { sedang, lemah }\end{array}$ \\
\hline 55 & 53 & $\mathrm{~L}$ & dewasa, laki-laki, & 80 & 78 & $\mathrm{P}$ & lansia, perempuan, \\
\hline
\end{tabular}




\begin{tabular}{|c|c|c|c|} 
& & & sedang, lemah \\
\hline $\mathbf{8 1}$ & 71 & $\mathrm{P}$ & $\begin{array}{c}\text { lansia, perempuan, } \\
\text { cepat, normal }\end{array}$ \\
\hline $\mathbf{8 2}$ & 42 & $\mathrm{P}$ & $\begin{array}{c}\text { dewasa, perempuan, } \\
\text { sedang, normal }\end{array}$ \\
\hline $\mathbf{8 3}$ & 47 & $\mathrm{P}$ & $\begin{array}{c}\text { dewasa, perempuan, } \\
\text { cepat, normal }\end{array}$ \\
\hline$\ldots$ & $\ldots$ & $\ldots$ & $\ldots$ \\
\hline
\end{tabular}

The process of grouping age based on the age range into the age group will use the data set as shown in the following table:

Table 5. Age Group Table

\begin{tabular}{|r|l|l|}
\hline No. & Age Group & \multicolumn{1}{c|}{ Age } \\
\hline 1 & Anak-Anak & $\leq 14$ thn \\
\hline 2 & Remaja & $15-24$ thn \\
\hline 3 & Dewasa & $25-60$ thn \\
\hline 4 & Lansia & $>60$ thn \\
\hline
\end{tabular}

\subsection{Data Transformation}

Medical data used in this study will be transformed first so that it is suitable for the data mining process.

Table 6. Transformation Data Table

\begin{tabular}{|l|l|}
\hline Atribut & Nilai \\
\hline $\begin{array}{l}\text { Kelompok } \\
\text { Umur }\end{array}$ & Anak-Anak, Remaja, Dewasa, \\
\hline Jenis Kelamin & 1 = Laki-laki, 0 = Perempuan \\
\hline
\end{tabular}

\subsection{Data Representation}

Data variables used in the information retrieval process are diagnosis, age group and gender. Examples of data used in this study are shown in the following table:

Table 7. Data Clustering Result for Man

\begin{tabular}{|c|c|c|c|c|}
\hline $\begin{array}{c}\text { Pat } \\
\text { ien } \\
\mathbf{t}\end{array}$ & $\begin{array}{c}\text { Age } \\
\mathbf{( O l d}\end{array}$ & $\begin{array}{c}\text { Gend } \\
\text { er } \\
(\mathbf{P} / \mathbf{L})\end{array}$ & $\begin{array}{c}\text { Blood } \\
\text { Pressure } \\
(\mathbf{m m H g})\end{array}$ & Item \\
\hline $\mathbf{1}$ & 44 & $\mathrm{~L}$ & 80 & $\begin{array}{c}\text { dewasa, laki- } \\
\text { laki, hipotensi }\end{array}$ \\
\hline & & & 143 & $\begin{array}{c}\text { dewasa, laki- } \\
\text { laki, } \\
\text { hepertensi1 }\end{array}$ \\
\hline $\mathbf{4}$ & 51 & $\mathrm{~L}$ & 149 & $\begin{array}{c}\text { dewasa, laki- } \\
\text { laki, } \\
\text { hipertensi1 }\end{array}$ \\
\hline $\mathbf{5}$ & 60 & $\mathrm{~L}$ & 149 & $\begin{array}{c}\text { dewasa, laki- } \\
\text { laki, } \\
\text { hipertensi2 }\end{array}$ \\
\hline $\mathbf{6}$ & 55 & $\mathrm{~L}$ & 161 & \\
\hline
\end{tabular}

\begin{tabular}{|c|c|c|c|c|}
\hline 8 & 50 & $\mathrm{~L}$ & 166 & $\begin{array}{c}\text { dewasa, laki- } \\
\text { laki, } \\
\text { hipertensi2 }\end{array}$ \\
\hline 10 & 33 & $\mathrm{~L}$ & 135 & $\begin{array}{l}\text { dewasa, laki- } \\
\text { laki, } \\
\text { prahipertensi }\end{array}$ \\
\hline 11 & 40 & $\mathrm{~L}$ & 170 & $\begin{array}{c}\text { dewasa, laki- } \\
\text { laki, } \\
\text { hipetensi2 }\end{array}$ \\
\hline 12 & 49 & $\mathrm{~L}$ & 134 & $\begin{array}{c}\text { dewasa, laki- } \\
\text { laki, } \\
\text { hipertensi1 }\end{array}$ \\
\hline 16 & 71 & $\mathrm{~L}$ & 146 & $\begin{array}{c}\text { lansia, laki- } \\
\text { laki, } \\
\text { hipertensi1 }\end{array}$ \\
\hline 17 & 67 & $\mathrm{~L}$ & 136 & $\begin{array}{l}\text { lansia, laki- } \\
\text { laki, } \\
\text { prahipertensi }\end{array}$ \\
\hline 18 & 78 & $\mathrm{~L}$ & 75 & $\begin{array}{l}\text { lansia, laki- } \\
\text { laki, hipotensi }\end{array}$ \\
\hline 19 & 62 & $\mathrm{~L}$ & 144 & $\begin{array}{c}\text { lansia, laki- } \\
\text { laki, } \\
\text { hipertensi1 }\end{array}$ \\
\hline 20 & 59 & $\mathrm{~L}$ & 135 & $\begin{array}{l}\text { dewasa, laki- } \\
\text { laki, } \\
\text { prahipertensi }\end{array}$ \\
\hline 21 & 52 & $\mathrm{~L}$ & 159 & $\begin{array}{c}\text { dewasa, laki- } \\
\text { laki, } \\
\text { hipertensi1 }\end{array}$ \\
\hline 22 & 72 & $\mathrm{~L}$ & 83 & $\begin{array}{l}\text { lansia, laki- } \\
\text { laki, hipotensi }\end{array}$ \\
\hline 23 & 78 & $\mathrm{~L}$ & 133 & $\begin{array}{l}\text { lansia, laki- } \\
\text { laki, } \\
\text { prahipertensi }\end{array}$ \\
\hline 24 & 68 & $\mathrm{~L}$ & 95 & $\begin{array}{l}\text { lansia, laki- } \\
\text { laki, normal }\end{array}$ \\
\hline 25 & 63 & $\mathrm{~L}$ & 135 & $\begin{array}{l}\text { lansia, laki- } \\
\text { laki, } \\
\text { prahipertensi }\end{array}$ \\
\hline 28 & 48 & $\mathrm{~L}$ & 79 & $\begin{array}{l}\text { dewasa, laki- } \\
\text { laki, hipotensi }\end{array}$ \\
\hline 32 & 67 & $\mathrm{~L}$ & 142 & $\begin{array}{c}\text { lansia, laki- } \\
\text { laki, } \\
\text { hipertensi1 }\end{array}$ \\
\hline$\ldots$ & $\ldots$ & $\ldots$ & $\ldots$ & $\cdots$ \\
\hline
\end{tabular}

Table 8. Clustering Result for Elderly Age

\begin{tabular}{|c|c|c|c|c|}
\hline $\begin{array}{c}\text { Pat } \\
\text { ien } \\
\text { t }\end{array}$ & $\begin{array}{c}\text { Age } \\
(\text { Old } \\
)\end{array}$ & $\begin{array}{c}\text { Gend } \\
\text { er } \\
(\mathbf{P} / \mathrm{L})\end{array}$ & $\begin{array}{c}\text { Blood } \\
\text { Pressure } \\
(\mathbf{m m H g})\end{array}$ & Item \\
\hline $\mathbf{1 6}$ & 71 & $\mathrm{~L}$ & 146 & lansia, laki- \\
\hline
\end{tabular}




\begin{tabular}{|c|c|c|c|c|}
\hline & & & & $\begin{array}{c}\text { laki, } \\
\text { hipertensi1 }\end{array}$ \\
\hline $\mathbf{1 7}$ & 67 & L & 136 & $\begin{array}{c}\text { lansia, laki- } \\
\text { laki, } \\
\text { prahipertensi }\end{array}$ \\
\hline $\mathbf{1 8}$ & 78 & L & 75 & $\begin{array}{c}\text { lansia, laki- } \\
\text { laki, hipotensi }\end{array}$ \\
\hline $\mathbf{2 2}$ & 72 & L & 83 & $\begin{array}{c}\text { lansia, laki- } \\
\text { laki, hipotensi }\end{array}$ \\
\hline & & & & $\begin{array}{c}\text { lansia, laki- } \\
\text { laki, } \\
\text { prahipertensi }\end{array}$ \\
\hline $\mathbf{2 3}$ & 78 & L & 133 & $\begin{array}{c}\text { lansia, laki- } \\
\text { laki, normal }\end{array}$ \\
\hline $\mathbf{2 4}$ & 68 & L & 95 & \\
\hline
\end{tabular}

2.7 Design of Monitoring System of Patient Health Development Based on Medical Record Using Fuzzy Logic Algorithm

The process of monitoring the health development of inpatients has two fuzzy functions or sets involved in it, namely:

a. Drug Usage Variable

Drug Use Variables have 3 fuzzy sets namely low, medium, high, as shown in figure below:

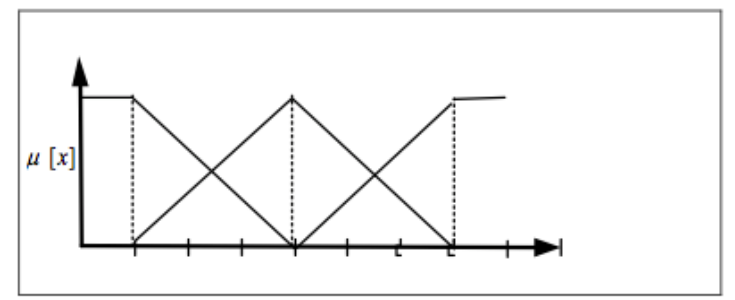

Figure 3. Drug Usave Variable Diagram

The low set uses the linear membership function approach down the left shoulder, the high set is the linear membership function approach up the right shoulder, while the set is using a triangular membership function approach. The following form of curve is the default curve of several factors that influence the use of drugs and their sets.

Membership Function :

$$
\mu_{\text {PakaiObatEENDAH }}[x]=\left\{\begin{aligned}
1, & x \leq 5 \\
\frac{15-x}{15-5}, & 5 \leq x \leq 10 \\
0, & x \geq 10
\end{aligned}\right.
$$

$$
\begin{aligned}
& \mu_{\text {PakaiObatEDANG }}[x]= \begin{cases}0, \quad x \leq 5 \text { atau } & x \geq 15 \\
\frac{x-5}{10-5}, & 5 \leq x \leq 10 \\
\frac{15-x}{15-10}, & 10 \leq x \leq 15\end{cases} \\
& \mu_{\text {PakaiobatIINGGI }}[x]=\left\{\begin{aligned}
0, & x \leq 10 \\
\frac{x-10}{15-10}, & 10 \leq x \leq 15 \\
1, & x \geq 15
\end{aligned}\right.
\end{aligned}
$$

P.S.: Numbers in the membership function formula for drug use represent the number of drug items used per day.

b. Treatment Duration Variable

The treatment duration variable has 3 fuzzy sets, namely: low, medium, high, as shown in figure below:

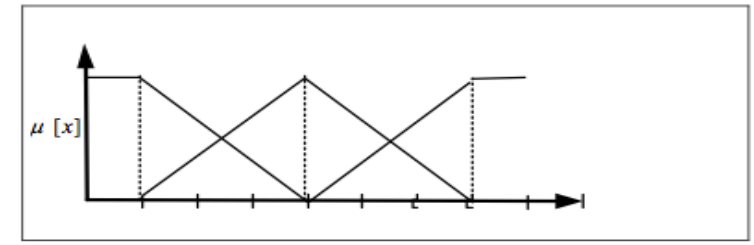

Figure 4. Treatment Duration Variable Diagram

The low set uses the linear membership function approach down the left shoulder, the high set uses membership the linear membership function approach rises the right shoulder, while the set is using a triangular membership function approach. The following form of curve is the default curve of several factors that influence the length of the diagnosis and its sets.

Membership Function:

$$
\begin{gathered}
\mu_{\text {LamaDiagnaaRENDAH }}[x]=\left\{\begin{array}{cc}
1, & x \leq 4 \\
\frac{12-x}{12-4}, & 4 \leq x \leq 12 \\
0, & x \geq 12
\end{array}\right. \\
\mu_{\text {LamaDiagnaaSEDANG }}[x]=\left\{\begin{array}{cc}
0, \quad x \leq 4 \text { atau } \quad x \geq 20 \\
\frac{x-4}{12-4}, & 4 \leq x \leq 12 \\
\frac{20-x}{20-12}, & 12 \leq x \leq 20
\end{array}\right.
\end{gathered}
$$




$$
\mu_{\text {LamaDiagnaaTINGGI }}[x]=\left\{\begin{array}{cc}
0, & x \leq 12 \\
\frac{x-12}{20-12}, & 12 \leq x \leq 20 \\
1, & x \geq 20
\end{array}\right.
$$

P.S.: Numbers in the duration treatment variable membership function represents the length of treatment in units of days.

Rule contains rules that apply to all events (combinations). This process functions to find a fuzzy output value from fuzzy input. The process is as follows: A fuzzy input value derived from the fuzzyfication process is then entered into a rule that has been made to be made into a fuzzy output. Following are the rules that will be used for fuzzy calculations:

[R1] IF Pemakaian obatTINGGI And Lama diagnosaTINGGITHEN

Kesehatan TIDAK MEMBAIK;

[R2] IF Pemakaian obatTINGGI And Lama diagnosa SEDANGTHEN

Kesehatan TIDAK MEMBAIK;

[R3] IF Pemakaian obatTINGGI And

Lama diagnosa RENDAHTHEN

Kesehatan TIDAK MEMBAIK;

[R4] IF Pemakaian obat SEDANG And Lama diagnosa TINGGI THEN

Kesehatan TIDAK MEMBAIK;

[R5] IF Pemakaian obat SEDANG And Lama diagnosaSEDANG THEN

Kesehatan TIDAK MEMBAIK;

[R6] IF Pemakaian obatSEDANG And Lama diagnosaRENDAH THEN

Kesehatan MEMBAIK;

[R7] IF Pemakaian obat RENDAH And Lama diagnosaTINGGI THEN

Kesehatan MEMBAIK;

[R8] IF Pemakaian obat RENDAH And Lama diagnosa SEDANGTHEN

Kesehatan MEMBAIK;

[R9] IF Pemakaian obat RENDAH And

Lama diagnosa RENDAH

THEN Kesehatan MEMBAIK;

Data clustering results that describe the relationship between length of treatment and patient tension can be seen in the following figure:

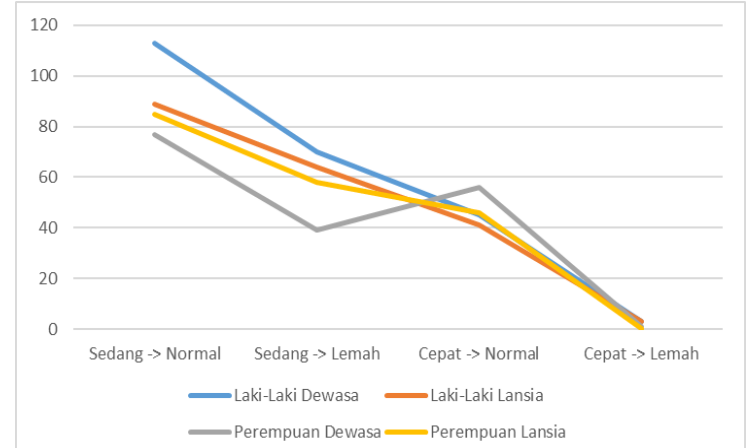

Figure 5. Comparison Chart of Clustering Result Data for Treatment Duration and Patient Tension

From the results of the clustering data obtained above, information can be obtained that if the duration of treatment is fast then the possibility of patient tension is weak, the percentage is very small. So that it can be said that if the duration of treatment is fast, then it is likely that the patient's tension is normal.

Data clustering results that describe the relationship between length of treatment and patient tension by grouping by patient sex can be seen in the following figure:

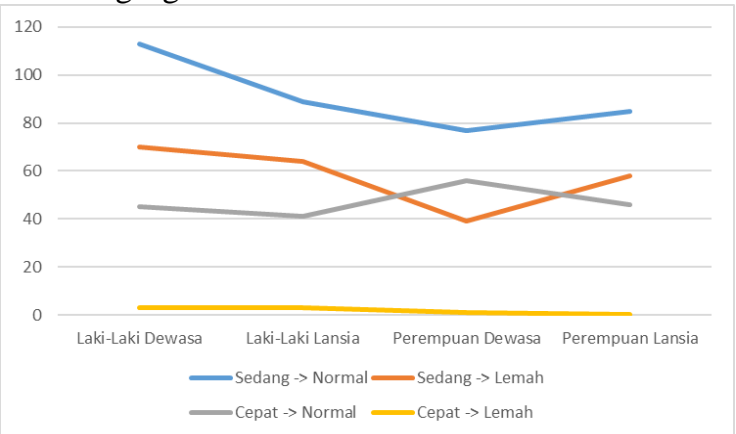

Figure 6. Comparsion Chart of Clustering Result Data for Treatment Duration and Patient Tension with Grouping Based on Patient Gender

From the results of the clustering data obtained above, information can be obtained that adult women have the highest recovery process success. In other words, it can be said that the process of caring for female patients has a higher success rate compared to male patients.

Meanwhile, the results of clustering that describe the relationship between gender and patient tension can be seen in the following figure: 


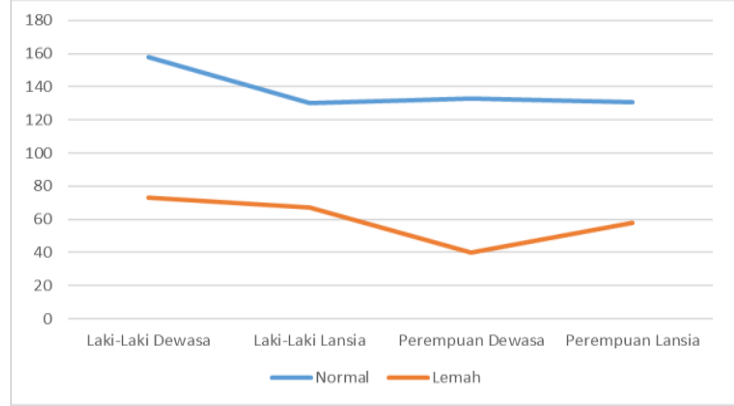

Figure 7. Comparison Chart of Clustering Result Data for Gender and Patient Tension

From the results of the clustering data obtained above, information can be obtained that the number of male patients whose weakness is far more than female patients. Meanwhile, the number of adult female patients whose weaknesses are the least.

Examples of the calculation of the value of $\mu$ for the number of drug use as many as 13 items and the length of treatment for 7 days can be seen in the following table:

Table 9. Computation Result $\mu$ on Treatment Duration and Drugs Usage Variable

\begin{tabular}{|c|c|c|c|c|}
\hline \multicolumn{5}{|c|}{ DRUGS USAGE } \\
\hline \multirow{4}{*}{$\begin{array}{c}\text { TREAT } \\
\text { MENT } \\
\text { DURATI } \\
\text { ON }\end{array}$} & & $\begin{array}{l}\text { Rend } \\
\text { ah: } 0\end{array}$ & $\begin{array}{c}\text { Seda } \\
\text { ng: } \\
0,4\end{array}$ & $\begin{array}{c}\text { Ting } \\
\text { gi: } \\
0,6\end{array}$ \\
\hline & $\begin{array}{l}\text { Rend } \\
\text { ah: } \\
0,62 \\
5\end{array}$ & 0 & 0,4 & 0,6 \\
\hline & $\begin{array}{c}\text { Seda } \\
\text { ng: } \\
0,37 \\
5 \\
\end{array}$ & 0 & $\begin{array}{c}0,37 \\
5\end{array}$ & $\begin{array}{c}0,37 \\
5\end{array}$ \\
\hline & $\begin{array}{l}\text { Ting } \\
\text { gi: } 0\end{array}$ & 0 & 0 & 0 \\
\hline
\end{tabular}

\subsection{Workflow Process Anlysis of Fuzzy Tsukamoto Method}

The working procedure stages of the Fuzzy Tsukamoto method in analyzing patient health can be described as shown in the following flowchart image:

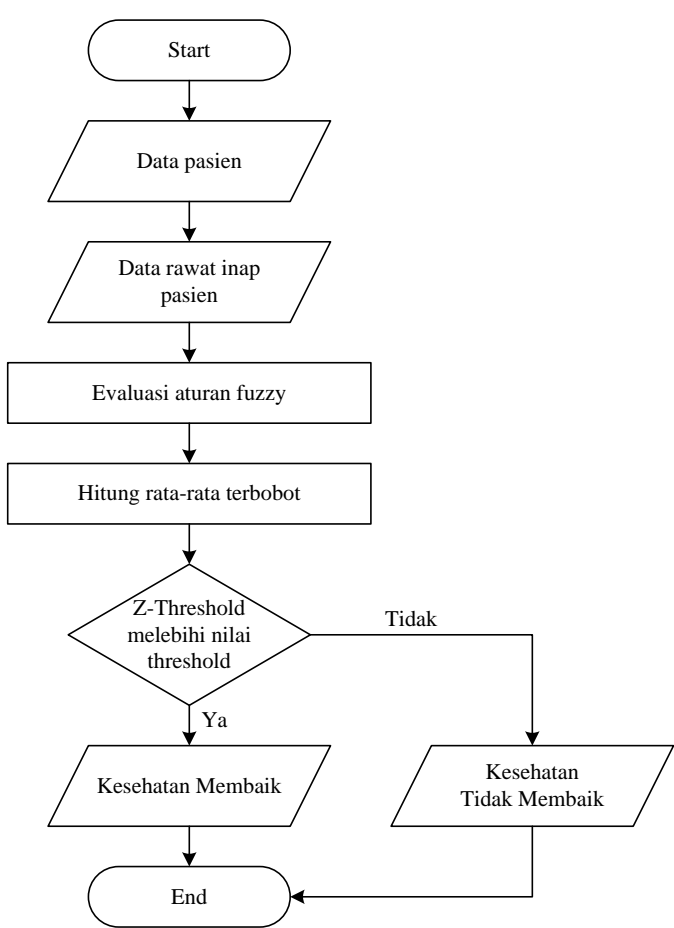

Figure 8. Flowchart Diagram of Fuzzy Tsukamoto Method

The process needs several data as input such as patient data and patient treatment record data. The data will be evaluated by using fuzzy rules and then it's checked whether the health condition is better or not.

\subsection{Diagram}

System work process design is used to show user interaction with the system made so that it can be known that the framework of this system is intended to know the outline of the system form that will be design. In the framework of this system framework, auxiliary tool use a use case diagram, as seen in Figure 7.

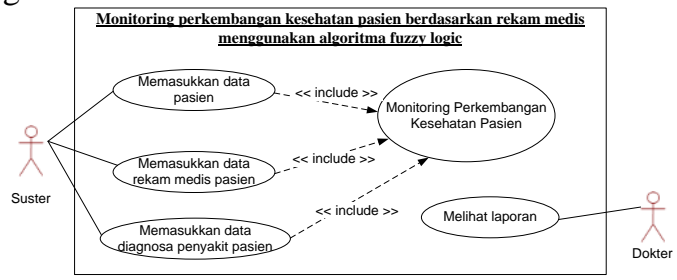

Figure 9. Use Case Diagram of Sytem

As shown ini figure 9, there are several process in system such as input patient data, input patient treatment record, input patient desease diagnosis, monitoring patient health improvement and showing report. 
Activity Diagram design of system could be seen as figure below:

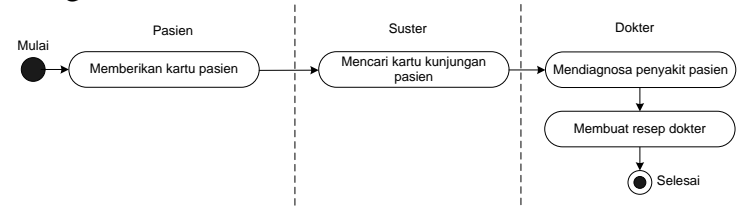

Figure 10. Activity Diagram of Proposed System

The medical record process starts with the patient giving the patient card to the nurse. After that, the nurse will look for a patient visit card. After the patient's turn, the patient will notify the complaint about the illness he has suffered to the doctor. After that, the doctor will diagnose the disease suffered by the patient and open a doctor's prescription to the patient.

\section{IMPLEMENTATION}

Display input from software includes:

1. Drugs Master, which could be used as a place for filling, changing and deleting drug data, as shown in figure 11 .

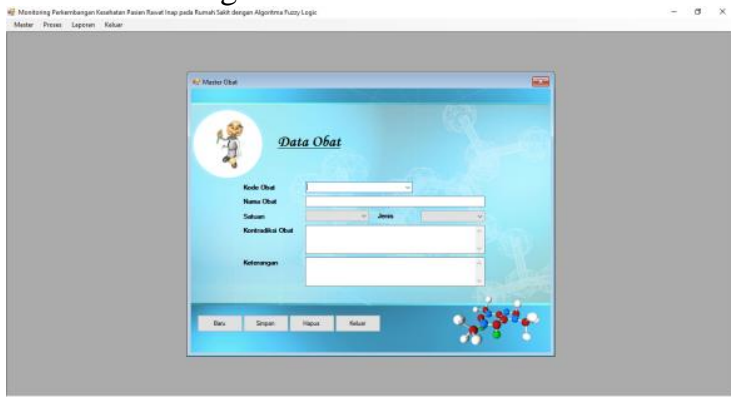

Figure 11. Drugs Master Form Display

2. Patient Master, which could be used as a place for filling, changing and deleting patient data, as shown in figure 12.

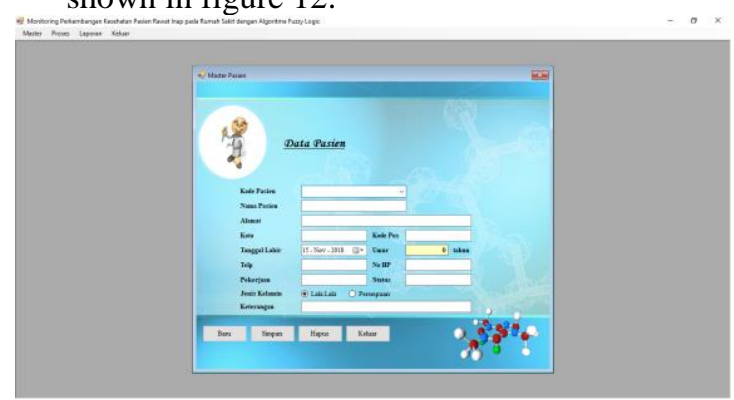

Figure 12. Patient Master Form Display

3. Desease Master, which could be used as a place for filling, changing and deleting desease data, as shown in figure 13 .

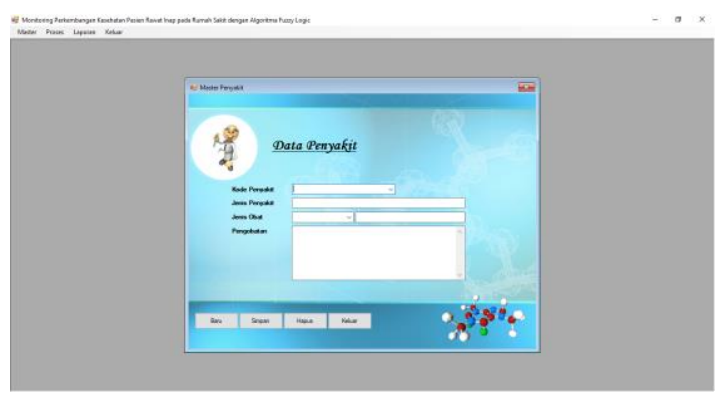

Figure 13. Desease Master Form Display

4. Health Development Monitoring Process, which serves to display the patient's health development results, as shown in figure 14 .

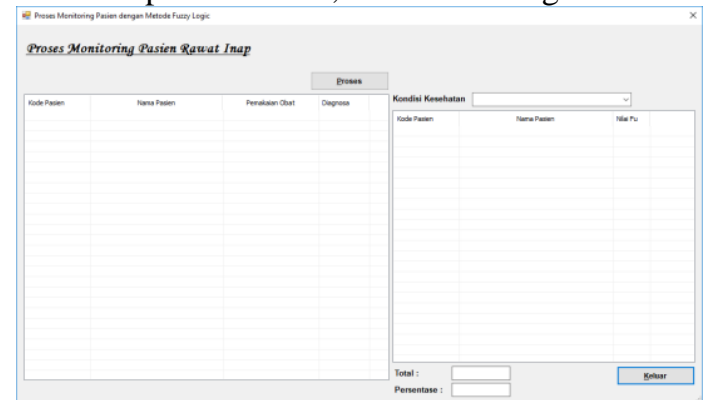

Figure 14. Health Development Monitoring Form Display

Based on the results of tests conducted on the system designed, it can be summarized in table form as follows:

Table 10. Monitoring Results of Patient Health Development

\begin{tabular}{|c|c|c|}
\hline \multirow{2}{*}{$\begin{array}{c}\text { Health } \\
\text { Condition }\end{array}$} & \multicolumn{2}{|c|}{ Patient Amount } \\
\cline { 2 - 3 } & $\begin{array}{c}\text { Real } \\
\text { Condition }\end{array}$ & $\begin{array}{c}\text { System } \\
\text { Monitoring } \\
\text { Result }\end{array}$ \\
\hline Improve & 256 & 202 \\
\hline $\begin{array}{c}\text { Started to } \\
\text { Improve }\end{array}$ & 125 & 154 \\
\hline Not Improve & 20 & 42 \\
\hline Get Worse & 5 & 8 \\
\hline
\end{tabular}

The process of testing the accuracy of the monitoring results obtained by the system will use the Confusion Matrix method as shown in the following description:

\begin{tabular}{|l|c|c|}
\hline & Predicted: NO & Predicted: YES \\
\hline Actual: NO & TN: 25 & FP: 0 \\
\hline Actual: YES & FN: 25 & TP: 356 \\
\hline
\end{tabular}

Result achieved:

Accuracy: $(\mathrm{TP}+\mathrm{TN}) /$ Total_data $=(356+25) / 406$

$=381 / 406=0.938(=93.8 \%)$ 
Error Rate: $(\mathrm{FP}+\mathrm{FN}) /$ Total_data $=(0+25) / 406$ $=25 / 406=0.062(=6.2 \%)$

\section{CONCLUSIONS}

After completing the design process of monitoring inpatient applications, some conclusions can be drawn such as the application is able to predict the number of patients whose condition is healthy, has improved or is still sick by using the Fuzzy Logic method and based on the results of the tests carried out, the information achieved is the accuracy of the application of the fuzzy logic method to the health monitoring process of patients in hospitals was $93.8 \%$, so the fuzzy logic method is suitable to be applied in the health monitoring process of patients.

\section{REFERENCES}

[1] Imaduddin Abdul Rahim, Oky Dwi Nurhayati, Kurniawan Teguh Martono, 2015, Pengembangan Aplikasi Sistem Cerdas untuk Menentukan Peminatan Jurusan di Universitas Diponegoro bagi Siswa Sekolah Menengah Atas, Jurnal Teknologi dan Sistem Komputer, Vol.3, No.2, April 2015 (e-ISSN: 2338-0403).

[2] Anita Dwi Wahyuni, MJ. Dewiyani Sunarto, Tan Amelia, 2015, Rancang Bangun Sistem Informasi Pos Pelayanan Terpadu pada Dinas Kesehatan Kota Surabaya Berbasis Web, JSIKA Vol. 4, No. 2. September 2015, ISSN 2338-137X.

[3] Salaman Rozak, Erwin Sutomo, Yoppy Mirza Maulana, 2018, Perencanaan Strategi Sistem dan Teknologi Informasi pada RS. Damian Lewoleba dengan Pendekatan Ward And Peppard, JSIKA Vol. 07, No. 05. Tahun 2018 ISSN 2338-137X.

[4] Fithriyah Andra, Hetty Ismainar, 2018, Produktivitas Penggunaan Waktu Kerja Perawat di Instalasi Rawat Inap Rumah Sakit Ahmad Yani Pekanbaru, Jurnal Kesmas, Volume 1, No 1, JanuariJuni 2018, e-ISSN : 2599-3399.

[5] Januari Nasir, Johnson Suprianto, 2017, Analisis Fuzzy Logic Menentukan Pemilihan Motor Honda Dengan
Metode Mamdani, Jurnal Edik Informatika Penelitian Bidang Komputer Sains dan Pendidikan Informatika V3.i2(177-186), ISSN : 2407-0491, EISSN : 2541-3716.

[6] Syaeful Anas Aklani, 2017, Metode Fuzzy Logic untuk Evaluasi Kinerja Pelayanan Perawat (Studi Kasus : RSIA Siti Hawa Padang), Jurnal Edik Informatika Penelitian Bidang Komputer Sains dan Pendidikan Informatika V1.i1(35-43), ISSN : 2407-0491, E-ISSN : 2541-3716.

Eka Kartika Untari, Alvani Renata Agilina, Ressi Susanti, 2018, Evaluasi Rasionalitas Penggunaan Obat Antihipertensi di Puskesmas Siantan Hilir Kota Pontianak Tahun 2015, Pharmaceutical Sciences and Research, 5(1), 32-39, 2018, ISSN 2407-2354.

[8] Haviluddin, 2011, Memahami Penggunaan UML (Unified Modelling Language), Jurnal Informatika Mulawarman, Vol. 6, No. 1.

[9] Han, J. and Kember, M., 2006, Data Mining Consept and Techniques, Second Edition, Morgan Kaufman, San Fransisco.

[10] Hendera, Sri Rahayu, 2018, Interaksi Antar Obat pada Peresepan Pasien Rawat Inap Pediatrik Rumah Sakit X dengan Menggunakan Aplikasi Medscape (Drug Interaction Between The Prescribing Pediatric Inpatients At Hospital $X$ Using Medscape Applications),

journal.umbjm.ac.id/index.php/jcps, Vol. 1 No. 2 (Maret, 2018), ISSN : 2598-2095

[11] Imam Sunoto, Ade Lukman Nulhakim, 2017, Mengukur Tingkat Partisipasi Pemuda Dalam Program Karang Taruna dengan Pendekatan Metode Fuzzy Infrence System Mamdani, Jurnal SIMETRIS, Vol 8 No 2 November 2017, ISSN: 2252-4983.

[12] Imran Dawy, Tian Songya, 2018, The Most General Intelligent Architectures 
of the Hybrid Neuro-Fuzzy Models, American Journal of Artificial Intelligence. Vol. 2, No. 1, 2018, pp. 1-6. doi: 10.11648/j.ajai.20180201.11.

[13] Irlando Moggi Prakoso, Wiwik Anggraeni, Ahmad Mukhlason, 2012, Penerapan Case-Based Reasoning pada Sistem Cerdas untuk Pendeteksian dan Penanganan Dini Penyakit Sapi, JURNAL TEKNIK ITS Vol. 1, No. 1(Sept. 2012), ISSN: 2301-9271.

[14] Nova Hasani Furdiyanti, Fania Putri Luhurningtyas, Ratna Sari, Yulianti, 2017, Evaluasi Dosis dan Interaksi Obat Antidiabetika Oral pada Pasien Diabetes Mellitus Tipe II, Jurnal Manajemen dan Pelayanan Farmasi, Volume 7 Nomor 4 - Desember 2017, pISSN: 2088-8139, e-ISSN: 2443-2946.

[15] Nunu Nurdiana, 2018, Perancangan Sistem Informasi Distribusi Obat Pasien Rawat Inap (Studi Kasus : RSUD Cideres Kadipaten), INFOTECH journal, Volume 4 Nomor 1 Tahun 2018, ISSN : 2460-1861

[16] Rizky Meimaharani, T. L., 2014, Analisis Sistem Inference Fuzzy Sugeno Dalam Menentukan Harga Penjualan Tanah Untuk Pembangunan Minimarket. Jurnal SIMETRIS, Vol. 5 No.1, 1-8.

[17] Robert Fuller, 1999, Fuzzy logic and neural nets in intelligent systems, in: $\mathrm{C}$. Carlsson ed., Information Systems Day, TUCS General Publications, Vol. 17, Turku Centre for Computer Science, $\mathrm{A}^{\circ}$ bo, [ISBN 951-29-1604-5], 1999 74-94.

[18] Ross, 2010, Fuzzy Logic with Engineering Application, New York: Mc-Graw Hill.

[19] Setya Haksama, M. Farid Dimyati Lusno, Diansanto Prayoga, M. Rifqo Hafidzudin Farid,Syadza Syahrah Shedyta, Syahrania Naura Shedysni, Sri Wiwoho Mudjanarko, 2018, Analisis Kinerja Unit Rawat Inap Rumah Sakit X Berdasarkan Kriteria Malcolm
Baldrige, JurnalSainHealth Vol. 2 No.2 Edisi September 2018, (C) Fakultas Ilmu Kesehatan Universitas Maarif Hasyim Latif Sidoarjo, p-ISSN : 2548-8333, eISSN : 2549-2586.

[20] Siti Marfu'ah, Liena Sofiana, 2018, Analisis Tingkat Kepatuhan Hand Hygiene Perawat dalam Pencegahan Infeksi Nosokomial, Kes Mas: Jurnal Fakultas Kesehatan Masyarakat, Volume 12, Issue 1, March 2018, pp. 29 37, ISSN: $1978-0575$.

[21] Sutojo, T., Edy Mulanto dan Vincent Suhartono. 2011. Kecerdasan Buatan, Penerbit Andi, Yogyakarta.

[22] Suyanto. 2011. Artificial Intelligence, Penerbit Informatika, Bandung.

Theresia Ratnadevi, Norisca Aliza Putriana, 2018, Pengaruh Pelayanan Farmasi Klinis di Rumah Sakit oleh Apoteker pada Kejadian Permasalahan Terkait Obat, SCIENTIA VOL. 8 NO. 1, FEBRUARI 2018， 8 (1) ; 104 - 118, 2018, p-ISSN : 2087-5045, e-ISSN : 2502-1834

Usman, Abdullah, 2017, Sistem Cerdas Diagnosa Penyakit dalam Menggunakan Jaringan Syaraf Tiruan dengan Metode Perceptron, JURNAL IPTEKS TERAPAN Research of Applied Science and Education V11.i4 (312- 322) Kopertis Wilayah X 312, ISSN: 19799292, E-ISSN:2460-5611.

[25] Vincent S. M. Tseng, Chao-Hui Lee, 2005, CBS: A New Classification Method by Using Sequential Patterns, Copyright by SIAM, Dept. Computer Science and Information Engineering National Cheng Kang University, Taiwan.

[26] Yasser M. Saber, Fahad Alsharari, 2018, Generalized Fuzzy Ideal Closed Sets on Fuzzy Topological Spaces in Sostak Sense, International Journal of Fuzzy Logic and Intelligent Systems, Vol. 18, No. 3, September 2018, pp. 161-166, http://doi.org/10.5391/IJFIS.2018.18.3.16 
1, ISSN(Print) 1598-2645, ISSN(Online) 2093-744X.

[27] Yue Wu, Biaobiao Zhang, Jiabin Lu \& K. -L. Du, 2014, Fuzzy Logic and Neurofuzzy Systems: $A$ Systematic Introduction, International Journal of
Artificial Intelligence and Expert Systems (IJAE), Volume (2) : Issue (2) : 2011.

[28]

Yunita, 2016, Penerapan Logika Fuzzy Dalam Sistem Pendukung Keputusan Pemberian Beasiswa BSM, Jurnal Techno Nusa Mandiri Vol. 42 XIII, No. 1 Maret 2016, ISSN 1978-2136 